\title{
Teilhabefokus und Soziologie sozialer Probleme. Eine Erkundung zu Forschungspotenzialen am Beispiel der Behinderungsfrage
}

\author{
Elisabeth Wacker
}

Eingegangen: 27. Juli 2020 / Angenommen: 16. Oktober 2020 / Online publiziert: 17. November 2020

(C) Der/die Autor(en) 2020

Zusammenfassung Teilhabeforschung hat Potenzial. Dies kann für das Feld der sozialen Probleme analysiert werden. Wie gesellschaftlicher Zusammenhalt in Verschiedenheit gelingen kann, wird hier am Beispiel der Vielfaltsdimension Behinderung systematisch reflektiert. Gerade mit Aufmerksamkeit für die Überschneidungen von Diversitätsmerkmalen wird dabei aufgedeckt, wie Behinderung als soziales Problem entsteht.

Diskurse um Diversität und Inklusion erweisen sich zusätzlich in der Ungleichheitsforschung als nützlich, weil sie die Diversitätsdimension Behinderung aus einem Schattendasein ins angemessene Licht rücken und zugleich den historisch gewachsenen festen Zugriff der Eingliederungshilfe auf dieses Handlungsfeld lockern. Zudem etablieren sich Diversität und Inklusion als sozialwissenschaftliche Trendsetter. Eine Abgrenzung des Teilhabekonzepts als relativ reale Realität von ihnen gelingt jedoch nur schwer.

In einem heraufziehenden Inklusions-Diversitäts-Dilemma kann Teilhabeforschung als Mediatorin agieren. Denn aus den andauernden Diskursen zur Teilhabe lassen sich Aspekte für planvolles Durchdenken und Gestalten ableiten. Passgenau in den jeweiligen Handlungsfeldern entsteht schließlich Teilhabe durch Teilhabe. Wichtig ist dabei zum einen eine verbindende kulturelle bzw. menschenrechtliche Perspektive: Alle sind gleich. Ebenso liegt die konstruktive Auseinandersetzung mit der Gegenwartsgesellschaft der Vielfältigen auf der Hand, denn alle sind verschieden, wobei schließlich drittens auch die Sicht auf Teilhabe inklusive zentral wirkt, denn sie lädt dazu ein, Abschied zu nehmen von Gleichschaltungsanliegen. Stattdessen fördert sie Formen der Gemeinschaft, in der Selbstbestimmung und Teilhabe, Vielfalt und Gleichwertigkeit, Inklusion und Exklusion als Entde-

\footnotetext{
E. Wacker $(\triangle)$

Lehrstuhl für Diversitätssoziologie, Fakultät für Sport- und Gesundheitswissenschaften, Technische Universität München, München, Deutschland

E-Mail: elisabeth.wacker@tum.de
} 
ckungszusammenhang in Teilhabefeldern verwoben sind. So gelangen Verfahren der Inklusion als genereller gesellschaftlicher Wandlungsprozess, als Strategie der Grenzüberschreitung und des Zutritts in Greifweite.

\section{Inclusion Focus and Sociology of Social Problems. An Exploration of Research Potentials by the Example of the Disability Issue}

Abstract Participation research is promising. It holds potential as a tool of analysis in the field of social problems. Using the disability dimension of diversity as an example, in this paper will be reflected systematically how social cohesion could be achieved in diversity. It is precisely by paying attention to the overlapping characteristics of diversity that disability is revealed to be a social problem.

Discourse on diversity and inclusion also proves to be useful in inequality research, because it brings the disability dimension of diversity out of a shadowy existence into the appropriate light and at the same time loosens the historically grown firm grip of rehabilitation assistance on this field. Moreover, diversity and inclusion are currently establishing themselves as social science trendsetters. Yet, delimiting the concept of participation as a relatively real reality, the distinction from them, has been difficult.

In a looming inclusion-diversity dilemma, however, participation research can act as a mediator. For, aspects for planned thinking and design can be derived from the ongoing debates on participation; in fields of social endeavor, participation perfectly tailored arises from participation. Here, a unifying cultural or human rights perspective is important: everyone is equal. Concurrently, a constructive examination of the contemporary society of the diverse is obvious, for all are different. Finally, including the view of participation also has a centering effect, for it invites us to bid farewell to concerns of conformity. Taken together, these three perspectives promote forms of community in which self-determination and participation, diversity and equality, inclusion and exclusion are interwoven as a context of discovery in fields of participation. Thus, we come closer to understanding inclusion as a general process of social change, for breaking boundaries and achieving access.

Die Idee sitzt gleichsam als Brille auf unsrer Nase, und was wir ansehen, sehen wir durch sie. Wir kommen gar nicht auf den Gedanken, sie abzunehmen.

Ludwig Wittgenstein - Philosophische Untersuchungen

\section{Teilhabe für jederman?}

Sie sprach von den kleinen Plätzen, der Welt der Einzelnen, den Nachbarschaften, den Bildungseinrichtungen und Betrieben, als sie am 10. Dezember 1948 die Verabschiedung der UN-Menschenrechtscharta begrüßte. Dort nämlich - so einst die Botschaft der Vorsitzenden der Menschenrechtskommission der Vereinten Nationen, Eleanor Roosevelt, - fangen die Menschenrechte an. Es ,,sind die Plätze, wo jeder Mann, jede Frau und jedes Kind gleiche Rechte, gleiche Chancen und gleiche Würde ohne Diskriminierung sucht. Solange diese Rechte dort keine Geltung 
haben, sind sie auch woanders nicht von Bedeutung. " ${ }^{11}$ Die Rede ist von einem vor mehr als 70 Jahren gegebenen Teilhabeversprechen an alle und überall und von der Suche nach seiner Umsetzung. Es geht um gerechte Chancen der Lebensgestaltung, die auch gegenwärtigen und zukünftigen Generationen garantiert sein sollen. Solchen Versprechen kann Forschung folgen und dabei Zusammenhänge von Teilhabe entdecken, ihre Wirkungen analysieren. sich auf die Spurensuche machen nach Menschenwürde, Lebensqualität und subjektivem Wohlbefinden. Ob diese ,Teilhabebrille“ auch der Soziologie der Sozialen Probleme gut zu Gesicht steht, wird hier gefragt. Können Erwartungen und Möglichkeiten über Teilhabeorientierung erfüllt werden, mit der gewünschten passenden „Differenziertheit“, feinkörnigen „Analysefähigkeit“ oder „Sensibilität“ (Die Redaktion 2006, S. 7), auch wenn Kategorisierungen ,spezifischer Randgruppen“ wie „Obdachlose, Alkoholiker, Gastarbeiter, entlassene Strafgefangene, Armut im Alter, Prostituierte, Behinderte“ problematisch bleiben (Groenemeyer 2006, S. $11 \mathrm{ff}$.)?

Dieser Spur wird hier am Beispiel der Diversitätskategorie „Behinderung“ nachgegangen. Als vor zwei Jahrzehnten Bendel (1999) der Soziologie Unaufmerksamkeit für das weit verbreitete soziale Differenzierungsmerkmal Behinderung attestiert, nennt er Pädagogik oder Medizin als Platzhalter. Eine „Wanderschaft“ zwischen Psychiatrie und Soziologie empfindet zehn Jahre später Finzen (2009, S. 1) nicht als anregend, sondern als eine ,unendliche (...) Geschichte frustrierender Begegnungen“, und er deklariert auch nach einer weiteren Dekade Normalität als eine der ungezähmten Kategorien in Psychiatrie und Gesellschaft (2018). Aus heutiger Sicht begibt sich soziologische Forschung dort also eher noch immer auf steiniges Gelände. Ein Blick in die Vergangenheit zeigt hier hingegen lebendige Aufmerksamkeit. In der qualitativ kulturorientierten Tradition der Chicagoer Schule waren funktionale Differenzierung, soziale Gemeinschaften und das Denken, Fühlen und Handeln in der spezifischen Welt der Kranken und der Psychiatrie etabliert. Eine Renaissance wäre also vorstellbar, womöglich Hand in Hand mit der Teilhabeforschung?

Aktuell formiert sich Teilhabeforschung vor allem im Feld der Rehabilitation. Sie verspricht die neue Spurensuche in gesellschaftlichen Handlungsfeldern, nach bewährten Leitideen von Demokratie, Partizipation, sozialer Gerechtigkeit und Lebensweltorientierung und will eine Befassung mit der Behinderungsfrage über medizinische Probleme und deren Folgen hinaus. Es solle vor allem auch um soziale Situationen gehen, komponiert aus biografischen, kulturellen und materiellen Lebensbedingungen von Personen mit chronischen Beeinträchtigungen sowie mit deren je eigenen Sinnkonstruktionen. Entsprechend rufen die Deutsche Vereinigung für Rehabilitation (DVfR) und die Deutsche Gesellschaft für Rehabilitationswissenschaften (DGRW) zur fachübergreifenden wissenschaftlichen Befassung auf. ${ }^{2}$

Hier ließe sich einhaken, und es ließen sich Muster sozialer Ungleichbehandlung in Verbindung von Behinderung und weiteren Merkmalen wie Alter, Geschlechtszugehörigkeit oder Hautfarbe zur kritisch-analytischen Betrachtung heranziehen. Weitere Forschungsdesigns sind denkbar. Im Rahmen einer Fellowship am Max-Planck-

\footnotetext{
1 https://www.dw.com/de/wo-fangen-menschenrechte-an/a-36586757. Zugegriffen: 20. September 2020.

2 Diskussionspapier Teilhabeforschung. Stand 15.11.2011. http://www.dgrw-online.de/files/paperteilhabeforschung-ef.pdf. Zugegriffen: 20. September 2020.
} 
Institut (MPI) für Sozialrecht und Sozialpolitik in München bestand die Chance, intensiv und über Fächergrenzen hinweg in den Programmlinien ,Wandel der Sozialsysteme und Teilhabe bei Behinderung“ (2010-2015) sowie „Dis[cover]ability \& Indicators for Inclusion“ (2015-2020) zu forschen und Wissenschaftlichen Nachwuchs zu qualifizieren. ${ }^{3}$ Die Inklusionsthematik wurde im Kontext von Behinderung im generellen gesellschaftlichen Wandlungsprozess aufgespürt, etwa im Sozialraum (Becker et al. 2013), im Erwerbsleben (Becker et al. 2015) und auch mit Blick auf ,Diversität \& Inklusion“ (Wacker und Groenemeyer 2014). Dem Merkmal „Behinderung“ wurde als soziale Praxis, d.h. „,doing disability“, als Handlungs- bzw. Leistungserwartung, als Institutionalisierung (kristallisierter Wissensbestand) sowie als Erfahrung in sozialer Praxis nachgegangen (Groenemeyer 2014), und es wurde verdeutlicht, dass es als Sammelkategorie für höchst verschiedene Bevölkerungsgruppen dient. Bio-psycho-sozial beeinträchtigt zu sein, entpuppt sich somit als unterschiedlich problematisierbares Problem und Frage der Deutung (Wacker 2013). Bis heute steht ein wissenschaftlicher Konsens zur Konstruktion von BeeinträchtigtSein und Behindert-Werden aus, bei gleichzeitiger gesetzes- und verwaltungstechnischer Operationalisierung und Nutzung. Zielführende Zweifel werden aber immer qualifizierter als Suche nach Teilhabe. Inzwischen ist diese Suche auch in einer repräsentativen Bevölkerungsbefragung konkretisiert. ${ }^{4}$ Damit kann Forschung mehr und mehr evidenzbasiert auf eine relativ reale Realität (Rosenthal 2017) blicken. So verdichten sich gegenwärtig gute Gründe, Behinderung keinesfalls als Eigenschaft einer Person, sondern als soziale Kategorie mit Teilhabebezug zu betrachten (Waldschmidt 2006), als

gesellschaftliches Produkt, das aus spezifischen gesellschaftlichen, kulturellen und materiellen Barrieren, die bestimmte Menschen an einer vollständigen Teilhabe an gesellschaftlichen Beziehungen und Ressourcen hindern, entsteht (Groenemeyer 2014, S. 164).

Behinderung bleibt so relevant im Diskurs um soziale Ungleichheit, wo sie lange ein Schattendasein fristete. Zugleich bahnen sich durch Teilhabeforschung eventuell Wege, die die Rehabilitationsforschung herausführen könnten aus einer (noch) bestehenden Dominanz von praktischen Anliegen der Eingliederungshilfe bzw. Förderbeschulung. ${ }^{5}$ Die Debatten um Ziele, Zuschnitt und Handlungsfelder zwischen Wissenschaft und Daseinsvorsorge sind lebendig. ${ }^{6}$,Wem ,gehört ${ }^{6}$ die

\footnotetext{
3 Die fachübergreifenden Forschungen sind u. a. dokumentiert in den Reporten des MPI (jüngst: Research Overview 2018. Max Planck Institute for Social Law and Social Policy [www.mpisoc.mpg.de]). Sie wurden begleitet und bewertet durch ein wissenschaftliches Kuratorium und über regelmäßige externe internationale wissenschaftliche Begutachtung.

4 Aktuell führt infas im Auftrag des Bundesministeriums für Arbeit und Soziales eine bundesweite Teilhabebefragung durch zu Zusammenhängen von Beeinträchtigung und erfahrener Benachteiligung in Haushalten und besonderen Wohnformen. Erhebungsinstrumente, wie Teilhabe erfahren wird bzw. Teilhabebarrieren erlebt werden, konnten in einer Vorstudie mit angemessener Vielfaltssensibilität für unterschiedliche Beeinträchtigungsformen und Lebensumstände entwickelt werden (Schäfers et al. 2016).

5 Hinz (2009) fordert dazu eine neue Differenzkultur.

6 Auch Leistungssysteme wie Altenhilfe oder Pflegesektor greifen inzwischen auf „Teilhabe“-Konzepte $\mathrm{zu}$.
} 
Teilhabe? Nutzen, Bereitstellung und Verfügbarkeit von Teilhabe als Allgemeingut“ stellt sich als Frage und Impuls für die affinen Forschungsfelder (Wacker et al. 2020). Die Aufmerksamkeitsspanne reicht dabei vom Bundesteilhabegesetz über Bundesteilhabeberichte mit Teilhabe als Programmziel (Wacker 2016) bis zur politischen Partizipation und theoretischen Versuchen, den unbestimmten Teilhabebegriff zu festigen.

Im Folgenden werden zunächst Potenziale und Risiken einer Teilhabeorientierung in der Forschung ausgelotet, aus der Perspektive der Diversitätsforschung und mit Blick auf Innovationspotenziale im Inklusions- und Intersektionalitätsfokus, um schließlich auf eine mögliche Kultur der Anerkennung einzugehen.

\section{Diversität, Inklusion und Teilhabeversprechen als Verlockung}

„Einfach machen“ ist seit mehr als einem Jahrzehnt der Teilhabeslogan der Bundesregierung. Dies genügt Forschungsanliegen nur bedingt. Vielmehr wird um theoretische Konzepte in der Ungleichheitsforschung seit langem ernsthaft gerungen. Denn Risiken, über ,unverbindliche Vielfaltsforschung“ bestehende vertikale Ungleichheiten wegzudifferenzieren, wegzupluralisieren, wegzuindividualisieren und wegzudynamisieren (Geißler 1994), werden seit langem kritisch gesehen. Das Individuum, das seinen Lebenslauf nun gestalten kann und muss, gerät zugleich schicksalhaft zum Maß von Erfolg oder Misserfolg des eigenen Lebens (Beck 1986). Als Anker bleiben zwar die Ansprüche auf Teilhabe an allen gesellschaftlichen Teilsystemen (Luhmann 1995, S. 139), die im Prinzip allen und überall Zugang gewähren müssen. Die Aufgabe, Teilhabeansprüche zu verwirklichen, fällt aber gänzlich den „Anspruchsberechtigten“ zu. Hier könnte Forschung zur Gehilfin werden und den Rechtfertigungsdruck auf Individuen mildern, indem sie lebensweltliche Vergemeinschaftungsformen und plurale Lebensstile erfasst. So aufgezeigte Muster sozialer Ungerechtigkeit und Vielfalt erlauben, sich nicht zwischen einem anhaftenden „Nicht-Können“ und klagenden „Nicht-Dürfen“ positionieren zu müssen. Stattdessen wird ein klarer (Mit-)Wirkungs- und Gestaltungs-Auftrag an Teilhabezielen an jeweilige Kontexte auf personaler und Umgebungsebene operationalisierbar. Forschung kann zeigen, ob gegebene Vielfach-Anrechte aller sich in reale Lebenschancen wandeln lassen, ob dabei systematisch Verliererinnen und Verlierer entstehen, z.B. weil vorrangig Agilität und Selbstvertretungskompetenz auf der Mikroebene Gatekeeper sind.

Entsprechend eingestimmt kann es spannend sein, affine Erfahrungsschätze und Fragen angrenzender Wissenschaftsfelder einzubinden. U.a. versprechen DiversityStudies, Inklusionskonzepte und Intersektionalität Potenzial. Es geht dort jeweils um Mehrdimensionalität und beobachtbare Zusammenhänge ungleich verteilter Chancen. Beispielsweise wurde die im Teilsystem Bildung in den 1960er-Jahren als prototypische Verliererin identifizierte „,katholische Arbeitertochter vom Land“ inzwischen vom „Migrantensohn bildungsarmer Eltern aus der Großstadt“ abgelöst (nach Gottburgsen und Gross 2012). Dies lässt sich über einen Intersektionalitätszugang nachvollziehen, und dabei kann die Verwobenheit der Vielfaltsdimensionen 
zum jeweiligen relevanten Forschungsgegenstand aufgedeckt werden. Soziale Probleme werden so sichtbarer, Benachteiligungslagen nicht vernebelt.

\subsection{Diversität und Unterschiede, die einen Unterschied machen}

„Macht der Differenz“ nennt Waldschmidt (2014) die Perspektive der Sozialstrukturanalyse, Intersektionalitätsforschung und Diversity Studies. Dabei ist nicht Teilhabe ihr Ansatzpunkt, sondern Diversität. Es geht um Normalitätsvorstellungen und Verantwortlichkeiten, jenseits der üblichen Aufzählung von Differenz-Merkmalen. Wer jeweils die „Anderen“ sind wird nämlich kulturell geklärt, gedeutet und bewerkstelligt. Ausgangspunkt kann das menschliche Tun sein, in dem sich wieder und wieder Modelle des Gewohnten verfestigen (Berger und Luckmann 1969) und dem ein Generalisiertes Anderes entspringt (Mead 1934).

Diversitätsforschung kann prinzipiell alle Formen der Unterschiedlichkeit zum Gegenstand erklären, häufig werden allerdings Gruppierungen in Zusammenhängen gewählt: etwa Jugendliche in einer alternden Gesellschaft, sexuelle Orientierung aus Sorge um gesellschaftliche (Un-)Ordnung, Herkunftsfamilie und Bildungschancen oder immer wieder Frauen als Abweichung von der männlichen Normalperson. ${ }^{7} \mathrm{Zu}-$ gleich hat der Diversitätsfokus im Humankapitel-Ansatz der Unternehmensführung Einzug gehalten. Dort werden systematisch Talente aufgedeckt, um aus der Vielfalt der Belegschaft Vorteile zu ziehen. Ähnliches intendiert ein normatives Diversitätskonzept im Bildungssektor, dem es um die gerechte Verteilung von Ressourcen geht (Haake 2018, S. 24). Das Bestreben „Schätze zu heben“ verbindet beide Ansätze und ermutigt im Spiel der Kräfte, bessere objektive Maßstäbe zu finden und weniger soziale Zuschreibungen zuzulassen. Diversitätsforschung agiert also auf persönlicher Ebene (mit Blick auf Begabung oder Kompetenz) und in Organisationen (bezogen auf Arbeitsverhältnisse oder Arbeitsplatzgestaltung). Zugleich verfolgt sie theoretische Bezüge (Intersektionalität, soziale Ungleichheit). Empirisch bewegt sie sich in verschiedenen Anwendungsfeldern (Kommunen, Unternehmen, Hochschulen) sowie im Diversity Management (Mensi-Klarbach et al. 2017, S. 4).

Als ein mögliches Traumpaar sozialwissenschaftlicher Befassung tritt nun Diversität in Verbindung mit Inklusion in Erscheinung. Den dynamischen Aufstieg belegt eine bibliometrische Analyse (1970 bis 2009): Das Themenfeld (Chancen-)Gleichheit stagniert und wird von Inklusion (inclusion) in der Publikationshäufigkeit überholt (Oswick und Noon 2014). Diversitätsforschung mit einer Inklusionsperspektive kann den tendenziell unbestimmten Diversitätsbegriff konkretisieren (Ferdman 2014, S. 4f.), zum Beispiel, wenn organisationale Inklusion nicht nur ,vermeintlich bedürftige“", sondern alle Mitglieder einer Organisation einbezieht und deren Bedürfnisse der Zugehörigkeit und Individualität thematisiert (Dobusch 2017, S. 60). Inklusion als Profil der Organisationskultur hat dann transformatorischen Charakter.

\footnotetext{
7 Goffmans Normalperson ist bekanntlich ,ein junger, verheirateter, weißer, städtischer, nordstaatlicher, heterosexueller protestantischer Vater mit Collegebildung, voll beschäftigt, von gutem Aussehen, normal in Gewicht und Größe und mit Erfolgen im Sport“ (1967, S. 158). Dieses symbolische Zentrum soll zwar laut Löw (2014, S. 32) verblassen, Muster und Maß der Intuition bleiben aber dennoch im Alltag auch nach 50 Jahren lebendig.
} 
Unterscheidungen zwischen passenden und abweichenden Organisationsmitgliedern verlieren Sinn, da alle als ,verschieden“ erkannt werden, und es daher gilt, konstruktiv mit Verschiedenheit umzugehen:

Inclusion can be less polemical and political than some other approaches - particularly those focused on ensuring representation, such as affirmative action, or those focused on specific group identities or ,,protected“ groups - but it does not negate or undermine those approaches: rather, it complements them and provides a lens and practices that can help make them more successful. (Ferdman 2014, S. 11 f.)

Im Mainstreaming-Ansatz konsequenter Inklusionsorientierung wird nämlich jenseits üblicher Gewährleistungslogiken für kategorisierte Gruppen - eine (breiten-)wirksame Haltung der Aufmerksamkeit gewonnen ebenso wie Praktiken der Wertschätzung von Diversität. Dieser ,inclusion turn" ${ }^{\text {"8 }}$ hat dynamisches Potenzial, meint Dobusch (2017, S. 62), die die Erschließung alternativer Forschungs- und Diskursräume erwartet. Damit wird Inklusionsforschung zur Innovationstreiberin.

\subsection{Inklusion als Eintritt ohne Anpassung}

$\mathrm{Zu}$ Fragen und Folgen der Inklusion bestehen unterschiedliche Wahrnehmungen und Interpretationen sowie eine Spezialität im deutschsprachigen Raum. Hier wird Inklusion häufig auf die gemeinsame Beschulung von Kindern und Jugendlichen mit und ohne Beeinträchtigung verkürzt. Im soziologischen Blick meint Inklusion ein Element gesellschaftlicher Analyse. Zudem lenkt unter dem Inklusionsbegriff weltweit die politische Programmatik unbedingter Zugehörigkeit zur menschlichen Gemeinschaft die Aufmerksamkeit. Forschung erhält somit unterschiedliche Anstöße und Lockrufe: Im Schulfokus ringt sie um Realitätsdeutungen und Realisierungsschritte, im wissenschaftlichen Diskurs kann sie Prüfstein der Reichweite und Relevanz von Fragefeldern sein, und weltweit trägt sie bei zur Suche und Analyse von Leistungsund Anpassungserwartungen. Die Menschenrechte fungieren dabei als transdisziplinäre „Leuchttürme“ für Suchende aller Länder. Zugleich bilden sich neue Abgrenzungen und finden sich unterschiedliche Auffassungen von ,drinnen und draußen“. Auch Inklusivität bleibt aber unbestimmt, Differenzierungen verstärken eher Exklusionseffekte, wie auch Luhmann einräumt (1997, S. 631). Was helfen dann multiple Teilhabeoptionen?

Forschung kann nun versuchen, Gleichheitsmaße (etwa einen Index of Inclusion) zu entwickeln, um „Ausschluss“ sichtbar zu machen. Einem Inklusionsgebot folgend ließen sich jeweils Maßnahmen planen und umsetzen, die Benachteiligung durch Ausgrenzung (Exklusion) reduzieren oder eliminieren. Ob dies bei diversen Gruppierungen mit Diversitätsprofilen gleiche Zugangsrechte, Zugehörigkeit und Zuweisungen über Zutrauen hervorbringt, steht dabei zur Debatte. Dass es auch um die ernsthafte Einbeziehung der Ausschlussgefährdeten gehen muss (partizipative Verfahren), zeichnet sich bereits ab. Dennoch bleibt die Gratwanderung zwischen

\footnotetext{
${ }^{8}$ Die Gruppe der deutschsprachigen Diversitätsforschung (D-A-CH) startet aktuell eine Buchreihe „Diversity and Inclusion Research“ bei Springer.
} 
Differenzkategorien und dem individuellen Recht auf Eigenart und Eigensinn (also Selbstbestimmung ohne Anpassungszwänge). Dies erfordert - wie der folgende Blick auf Intersektionalität zeigt - komplexe Arrangements (Krell 2014). Zugleich entstehen Wertungen der jeweils erfassten „Abweichung“ (Differenz).

Lösungen werden gesucht (Wacker 2014, S. 240 f.). Bereits seit 70 Jahren (Marshall 1950) gibt es Wegweisungen in soziologischer Denkweise mit dem Konzept der „citizenship“ (Bürgersein). Parsons (1972) trug in seiner Gesellschaftstheorie dazu bei und auch Luhmann (1980) nutzte den Inklusionsbegriff für die Beschreibung einer Strategie der Grenzüberschreitung und des Zutritts in gesellschaftliche Teilsysteme. Denn:

Jede Person muß ... Zugang zu allen Funktionskreisen erhalten können ... Das Prinzip der Inklusion ersetzt jene Solidarität, die darauf beruht, daß man einer und nur einer Gruppe angehört. (Luhmann 1980, S. 4)

Faktisch treten jedoch Neben- bzw. Wechselwirkungen auf, denn: „Die Exklusion aus einem Funktionsbereich verhindert die Inklusion in andere“ (Luhmann 1980, S. 584).

Auch wenn mit Inklusion Einpassungsanforderungen entfallen, bleiben also Risiken, die sozialer Zusammenhalt nicht ,einfach“ heilt. Deswegen sorgt sich Nassehi (2014, S. 57) in Bezug auf einen bürgerlichen Diskursstil, der auf zivilisierte Weise mit Vielfalt umgehen könne und pluralistische Lebensformen eröffne, zugleich (ganz im Stil der angerufenen Diskurse), ob man so etwas wie Zusammenhalt als „die andere Seite von Vielfalt“" politisch tatsächlich herstellen und auf diesem Wege Teilhabehindernisse beseitigen könne.

Im Bereich der Eingliederungshilfe bei Behinderung kämen mit einem Inklusions-Index insbesondere Leistungssysteme unter dem Konzept von Rehabilitation und Teilhabe auf den Prüfstand, die Inklusion exklusiv konstruieren und damit Sonder-Realitäten schaffen, ohne verwirklichte Teilhabe im oben genannten Sinn zu intendieren (Wacker et al. 2009).

\subsection{Teilhabeanspruch, Interdependenz und intersektional verbundene Vielfalt}

Auf den Kitt der Gesellschaft als stillschweigende Allianz zwischen „Normalen und Stigmatisierten“ machte bereits Goffman (1967, S. 160) aufmerksam. Je genauer man sich nun hinzusehen erlaubt, um so erkennbarer werden Kompromisslinien, die Vielfalt verbinden. Sozialarbeit kann mit Teilhabemaßen, also Art und Umfang von verwirklichter Teilhabe, statt mit Identitäts- und Zugehörigkeitskategorien, im Prinzip aufdecken, wie Diversität einen Unterschied macht (Mecheril und Plößer 2018, S. 283), ohne eine „dauerhafte Defizienz“ vor- und zuschreiben (Kaufmann 2012, S. 1285). Stattdessen könnte aus einer Merkmalsperspektive Ohne-Ordnung (also über Nicht-Zuordnung) die neue sozialstaatliche Normalität der Vielfalt hervorgehen. Wie später bedacht wird, sollten Gestaltungsaufgaben partizipativ gelingen. Aus Forschungsperspektive hilft ein Verständnis von Intersektionalität, d.h. dafür, wie sich Vielfalt und Teilhabeansprüche faktisch zueinander verhalten.

Als „Prophetin“ von Intersektionalität gilt die us-amerikanische Juristin Kimberlé Crenshaw (1989, 2010). Intersektionalitätsforschung nutzt den Begriff der Interde- 
pendenz, um soziale Ungleichheit zu beobachten und zu beschreiben. Es geht dabei nicht alleine um eine Perspektive der Diversität, sondern darum, ungleiche Machtverhältnisse methodisch zu klären (Walgenbach 2018, S. 41).

Bei der Frage nach Behinderung in einer alternden Gesellschaft werden beispielsweise vielfache Interdependenzen aufdeckbar. Differenzen, Überschneidungen und Wechselbeziehungen zwischen den Merkmalen Behinderung und Alter machen einen Unterschied: Etwa wann im Lebensverlauf eine dauerhafte Beeinträchtigung eintritt, welcher Art und Ausprägung sie ist, aber ebenso, wie Wahrnehmungen sich dann in typisch weiblichen Biografien ausprägen, als Element der Strukturen der sozialen Erfahrung (Walthes und Hitzler 2018, S. 11). In ,gefrorenen“ Pragmatiken und institutionellen Zuweisungen (z.B. zur Mutterschaft) setzen sich zugleich Benachteiligungen und Erwartungen fort (Schönig 2019, S. 198). Mit einer Fürsorge- oder Versorgungsbrille zeigen sich andere Ergebnisse als bei der Suche nach Selbstbestimmung und Teilhabe. So wird die Positionierung nach Mustern der Diversität und Inklusion möglich und wohl auch wissenschaftstauglich. Eine Liaison von Intersektionalität und Diversität wird hierzu vor allem in den Diversity Studies diskutiert. Walgenbach (2018, S. 34 ff.) warnt aber vor einer einseitigen Verlockung, da es den Diversitätsanliegen an der gebotenen Neutralität mangle. Intersektionalität würde womöglich zum Werkzeug degradiert, um Wechselwirkungen aufzudecken oder gar zur Handlangerin, die nutzbare (und unnütze) Arbeitskraft entdeckt. Dem solle man mit dem Paradigma (!) $)^{9}$ der Intersektionalität entgegentreten. Keinesfalls sollten soziale Gruppen gegeneinander ausgespielt werden und Diversität zum beliebigen Aushängeschild verkommen (Walgenbach 2018, S. 37). Die Besonderheit des Diversity Paradigma (!) sei gerade, parteilich zu sein, zugunsten der Potenziale von Diversität. Schöer (2015) schlägt - versöhnlicher - einen Dreiklang aus Diversity Management (mit Erfassen von Defiziten), Intersektionalität (mit Bewertung von Kompetenzen) und interkultureller Öffnung (mit Chancen auf gelebte gesellschaftliche Teilhabe) vor.

\section{Anerkennung im Teilhabe-Diversitäts-Dilemma und Transformationspotenzial der Teilhabeforschung}

Teilhabeforschung nutzt Lebensweltorientierung und zumeist qualitative Forschungszugänge als Blickwinkel, aber ebenso als Objektdetails. ,Teilhabe-Wahrheiten“ sind bottom-up, aus einer Froschperspektive, oder top-down, aus einer Vogelperspektive beschreibbar. Jeweilige Blickwinkel multiperspektivisch umzusetzen, ohne sich in Komplexitäten des Alltags zu verlieren, ist anstrengend, womöglich

\footnotetext{
9 Ob diese Debatte im Kuhnschen Sinne (Kuhn 2009) den Begriff Paradigma verdient, ließe sich fragen.
} 
auch aporisch. ${ }^{10}$ Genau dort aber kann Teilhabeforschung verlockende Routinen aufbauen, die in konkrete Handlungsaufträge wandelbar sind.

Im Herbst 2019 fand der 1. Kongress der Teilhabeforschenden an der HumboldtUniversität in Berlin statt. Das junge Forschungsfeld formiert sich bereits seit einigen Jahren in den deutschsprachigen Ländern. Profilbildend sind Lebenslagen- und Lebensweltorientierung, verbunden mit partizipativen Methoden. Im transdisziplinären Zuschnitt ist Teilhabeforschung - wie oben angesprochen - auch anschlussfähig zu neuen Forschungs-Communities (wie der international etablierten DiversityForschung, One-Health-Forschung oder den Disability-Studies). Diese verbindet, besonders aufmerksam zu sein für Alltag und Vielfalt in Gemeinschaften oder Bevölkerungen. Notwendige Strukturentwicklungen für Zusammenhalt, ebenso aber Prozesse von Zugehörigkeit einzelner und Gemeinschaftsbildung werden einbezogen.

Bislang befasst sich die deutschsprachige Teilhabeforschungsgemeinschaft vorrangig mit Behinderung, Betroffenheit, Selbst-Identifikation und (neuer) politischer Partizipation. Aus einer Diversitätsdimension wäre sie daher Nischenforschung. Zugleich zeigen sich aber Potenziale für eine sich profilierende Transformationsforschung, die Wege zu einer inklusiven Gesellschaft bahnen will und dabei Diversität als grundlegenden Bauplan beachtet und anerkennt (Wacker 2019).

Das Veränderungspotenzial, ein Nicht-Weiter-So!, soll im Folgenden näher bedacht werden. Entsprechende Anliegen der Innovation und Disruption ließen sich möglicherweise auch mit bewährten Verfahren der Aktionsforschung als Forschung mit Handlungsgebot (Lewin 1948) umsetzen, in kultureller Perspektive, mit neuem Gespür für eine Gesellschaft der Vielfalt und auf der Fährte des Wandels zur inklusiven Gesellschaft.

\subsection{Die kulturelle Perspektive: alle gleich I Human Rights Approach}

Im menschenrechtlichen Blickwinkel wird das Teilhabeanliegen mit der generellen Frage verbunden, wie Menschen in der Gesellschaft der Gegenwart zueinanderstehen. Mit der Aufmerksamkeit für gleiche Rechte aller Menschen rückt Behinderung in die Mitte der Gesellschaft. Dies umfasst auch, nach der Verfügung über gesellschaftlich relevante Ressourcen wie Macht, Bildung und Einkommen zu suchen, die das menschenrechtliche ,alle gleich“ operationalisieren. Hier lenkt die seit einer Dekade rechtlich und politisch wirksame Behindertenrechtskonvention der Vereinten Nationen (UN-BRK: United Nations 2006) die Aufmerksamkeit. Belange von Menschen mit Beeinträchtigungen ebenso wie deren Selbstbewusstsein und Chancen werden zunehmend gesehen. Dennoch finden sie nicht immer Gehör, viele Fragen

\footnotetext{
${ }_{10}$ Die Frage ist, wie man mit unlösbaren theoretischen Problemen umgeht: Einerseits ist die paradoxe Erkenntnis des eigenen Nichtwissens auf der Suche nach Wahrheit möglich, andererseits lassen sich Aufgaben mit einem Untersuchungsdesign definieren, das Durchdenken und Diskurs planvoll ermöglicht. Fragt man sich etwa in der Welt der Medien: „Warum passiert eigentlich jeden Tag genau so viel, dass die Zeitung voll wird?" (Kocks 2001, S. 217), ist ein Lösungsweg, zwischen dem An-Sich-Faktischen und dem FürSich-Faktischen zu unterscheiden. Geht es um Teilhabefragen unterstützt entsprechend eine Perspektive, die nicht unspezifisch die weltweite Teilhabe an sich, sondern in Bezug auf spezifische Handlungsfelder oder Ungleichheitskonstellationen betrachtet.
} 
und Wünsche bleiben offen. Dies versucht Teilhabeforschung evidenzbasiert aufzudecken und dabei auf Wirkungen bzw. Unterschiede zwischen Recht und Realität zu achten. Ziel ist zunächst, Teilhaberisiken möglichst treffsicher und differenziert sichtbar zu machen.

Menschenrechte zu haben genügt allerdings nicht, es geht - wie bei der Teilhabe generell - um Verwirklichungschancen (entsprechend dem Denkmodell des Capability Approach I Befähigungs-Ansatz nach Sen 1985). Ob unabhängig von einer Beeinträchtigung jede Person in ihrem Lebenskreis ihre Anliegen im Lebenslauf in Wirklichkeiten ummünzen kann (und dies weitgehend selbstbestimmt), ist der passende Maßstab. Mit der Frage nach verfügbaren Ressourcen lässt sich zugleich der Ermöglichungsspielraum verdeutlichen, den Settings oder Maßnahmen in sich tragen.

Weil sich Teilhaberisiken und -limitierungen in spezifischen Handlungsfeldern finden, in denen Personen mit ihren jeweiligen Beeinträchtigungen z. B. auf bauliche Barrieren, rechtliche Zugänge, kulturelle Einordnungen etc. treffen, lassen sich hier ggf. Benachteiligungen aufdecken. Mit entsprechend solidem Wissen sollen Risiken vermieden oder reduziert werden. Dazu differenziert, beobachtet und bewertet Teilhabeforschung in der UN-BRK-Logik (United Nations 2006) nach acht Teilhabebereichen: Bildung und Ausbildung, Erwerbstätigkeit und materielle Lebenssituation, Alltägliche Lebensführung, Gesundheit, Freizeit, Kultur und Sport, Sicherheit und Schutz der Person, Politische Beteiligung sowie Familie und soziales Netz.

Nach einem Lebenslagenansatz soll der Spielraum beurteilt werden, der sich Menschen bietet, um ihre Grundanliegen bei der Gestaltung des eigenen Lebens zu erfüllen (Weisser 1978). Über eine Perspektive der Gleichwertigkeit kommen Sozialstruktur und Soziale Ungleichheit als potenzielle Spielraumgeber - wie im oben genannten Capability Approach (Sen 2010,2007) in den Fokus. Teilhabeforschung macht also Veränderungsbedarf sichtbar und berücksichtigt auch komplexe Unterstützungsbedarfe.

Weil Teilhabeforschung den Anspruch erhebt, Heterogenität zu beachten, betrachtet sie Persönlichkeitsmerkmale einzelner ebenso wie gesellschaftliche Einbettungen und Verflechtungen (Gardenswartz und Rowe 2003). Niemand soll auf nur ein Merkmal reduziert werden, Binnendifferenzierung und Zuordnungen werden entsprechend ausgeformt und mit den Gleichbehandlungsgesetzen in Verbindung gebracht (Wacker et al. 2009). Dennoch bleiben viele Fragen offen, beispielsweise, ob eine Angleichungsstrategie eher Gerechtigkeit hervorbringt, wenn auch um den Preis steigender Diskriminierungsrisiken? Ob die Annahme einer fiktiven Gleichheit zwar verkürzt ist, aber eine bessere Praxis ermöglicht, weil es um Freisetzung von Chancengerechtigkeit geht und daher als berechtigt, wahrgenommen, gehört und beteiligt zu werden, Priorität haben muss?

Hierzu Abwehrrechte, Gleichbehandlungsgebote und Teilhabeaufgaben zu prüfen, erfordert forschungsmethodisch ein Kaleidoskop von hoher Aufmerksamkeit, viel Verständnis und gesteigerter Flexibilität. Auch die Grundsatzfrage bleibt zu lösen, ob Verschiedenes gleichbehandelt werden soll oder ob es vor allem darum geht, Ungerechtigkeit abzubauen und mit Vielfalt konstruktiv umzugehen. Diese bis dato am Beispiel von Behinderung aufgegriffenen Fragen stellen sich - in einer 
selbstreflexiven Anstrengung - auch generell zu Sozialen Problemen, idealerweise in wechselseitiger Abstimmung mit anderen Forschungscommunities.

Soweit ein erster Zwischenstand aus dem Blickwinkel „Suche nach Teilhabehindernissen“. Es geht um Forderungen nach Chancengerechtigkeit (zwischen Equity und Equality), nach Gleichstellung (als Angleichungsstrategie mit Gleichheitsrechten, Anti-Diskriminierung, Nachteilsausgleichen I Affirmative Action) und nach Ausgleich als Mainstreaming-Ansatz gegen strukturelle Benachteiligung aller Bürgerinnen und Bürger. Im Folgenden soll nun spezifisch die Vielfaltsgesellschaft den Blickwinkel vorgeben.

\subsection{Die Ressourcenperspektive: alle verschieden I Human Resource Approach}

Wenn es gerecht wäre, jeder und jedem das Gleiche zu geben, wäre die Aufgabe leicht lösbar. Stattdessen weist Teilhabeforschung auf grundlegende Spannungsverhältnisse und Verflechtungen von Diversität und Inklusion hin. Dabei begegnet sie den Diversity-Studies, auch in ihrer leistungsbezogenen Ausformung, mit dem Fokus auf Human Resource.

Menschliche Kräfte entwickeln und entfalten sich in der Lebensspanne und in jeweiligen gesellschaftlichen Teilsystemen; dort liegen auch Treiber für Umgestaltungen in Wissenschaft und Gesellschaft. Voraussetzung sind zunächst die Wahrnehmung und Überzeugung von der Verschiedenheit der Menschen, gefolgt von Strategien und der geeigneten Systematisierung des Umgangs damit.

Die Praxis im Vielfaltsbezug bleibt hindernisreich, wie dies Edward Twitchell Hall (1976) mit seinem Eisberg-Modell der wissenschaftlichen Öffentlichkeit vorstellte. Unter dem Titel „Beyond Culture“ deckt er Unsichtbares in einer Gesellschaft auf. Wie bei einem Eisberg zeigt sich nur die Spitze (etwa Alter, Geschlecht, Sprache). Unter der Oberfläche verborgen liegen jedoch viele relevante und schwerwiegende Tatbestände, die sich der ersten Wahrnehmung entziehen. Das nicht Sichtbare, scheinbar fraglos Gegebene, wie alltägliche Wissensvorräte und Zugänge zu allen sozialen, kulturellen und wirtschaftlichen Prozessen, gilt es nun zu entdecken und dann für gleiche Rechte, aber ungleiche Chancen zu sensibilisieren. Denn das Unconscious Conscious (Un-Bewusstsein) durchdringt Human Resource (HR), Werte, aber auch Ungleichheitsforschung und Reflexivität. Erkundungsstudien zeigen die Macht „vorhandener Wissensvorräte“ bei Fragen von Teilhabe und Behinderung, beispielsweise bei geschulten Beratenden und Lehrenden im Bildungssektor (Demant 2017), also genau dort, wo die Chancen auf Teilhabe an Bildung ,vergeben“ werden, und ebenso beim HR-Fachpersonal in öffentlichen und privaten Organisationen, das ,gate keeping“ in die Arbeitswelt verkörpert (Dobusch 2015).

Wirksame Forschung zur Aufdeckung dieses Verborgenen kann nicht auf eine Verhaltensebene beschränkt sein, sondern muss vor allem die Verhältnisebene erreichen. Deswegen ist der Blickwinkel ein Bezug zu Lebenssituationen und Lebenslagen. Bei Behinderung geht es auch um Lebensumstände in Obhut der Wohlfahrtspflege und/oder Eingliederungshilfe, die mit weit über hundertjähriger Tradition bis heute ihren Fürsorgeauftrag wahrnehmen.

Hall richtet seinen Aufklärungsruf vor beinahe einem halben Jahrhundert an die Fachwelt und lädt zur aktiven Teilhabe ein, um bestehende Alltagskulturen mit spe- 
Abb. 1 International Classification of Functioning, Disability and Health (ICF) (WHO 2001)

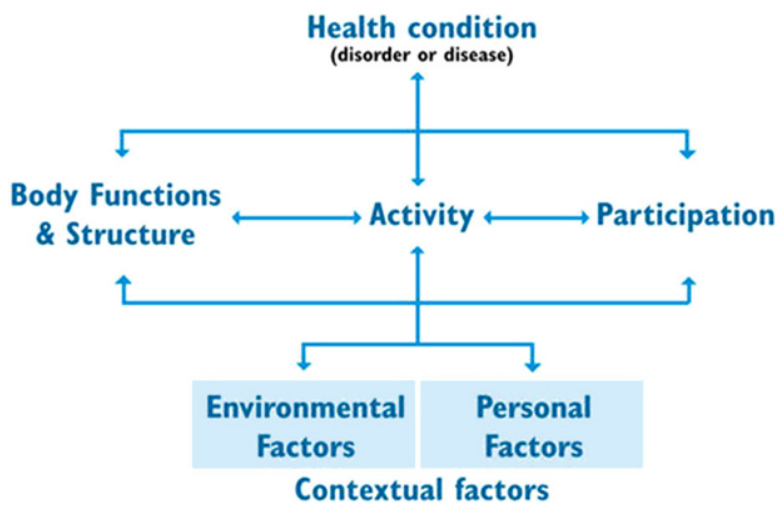

zifischem Wissen anzureichern. Entdeckungen des Verborgenen sollen „by doing“ gelingen. Damit sind Menschen nicht nur Untersuchungsgegenstände, sondern relevant verwoben in ihre jeweiligen Kontexte, die materielle und soziale Welt. Deswegen bieten sich Methoden der Aktionsforschung an, ebenso wie partizipative Verfahren mit der reflexiven ,awareness“ für Beeinträchtigungen und Behinderung, die die „International Classification of Functioning, Disability and Health“ (ICF) (WHO 2001) ${ }^{11}$ bietet (Abb. 1).

Denkbare „Inclusion Indices“ sind im Grundsatz nichtdiskriminierend, es sei denn sie normieren und bewerten Vielfalt. Sie sind nützlich, um Diskriminierung nicht mehr als Einzelschicksal darzustellen, sondern als Ordnungsfaktor. Erscheint zugleich Verschiedenheit als normal, geht es nicht mehr darum sie zu beseitigen, sondern darum Vielfalt nicht $\mathrm{zu}$ benachteiligen. Einbeziehung aller in Teilhabeforschung fußt auf der erwähnten Lebensweltorientierung (Schütz und Luckmann 1975, S. 23), die vorliegende objektive Wissensvorräte anreichert um die Bedeutung und Wirkung für subjektiv wahrgenommene Lebensqualität und Wohlbefinden. Die Kette der Selbstverständlichkeiten kann durchbrochen werden. Dies wäre Aufgabe und Stärke zukünftiger Teilhabeforschung, wenn sie Transformationswissenschaft sein will.

Die nächste Zwischenbilanz lautet folglich: Vielfalt zu erkennen und mit ihr konstruktiv umzugehen ist wesentlich. Dies gilt in allen Lebensbereichen und Lebensphasen. Eigene Ressourcen sollen sich überall entfalten können, in Bildung, Arbeit, Wohnen, Sicherheit, Mobilität, Gesundheitsversorgung, Freizeit etc. Eine

\footnotetext{
${ }_{11}$ Die ICF ist der maßgebliche internationale und fachübergreifende Standard, um Gesundheit und Behinderung bzw. Beeinträchtigungen einer Person (individuelle Ebene) oder in einer Bevölkerung (gesellschaftliche Ebene) zu erfassen. Sie beleuchtet im Alltagsbezug die fließenden Grenzen zwischen Gesundheit und Behinderung (salutogenetischer Ansatz: Antonovsky 1997) und verdeutlicht die Breite der Beeinträchtigungserfahrungen als Element des Menschseins (,,mainstreams the experience of disability and recognizes it as a universal human experience"). Behinderung wird also mehrdimensional betrachtet mit Bezug zu verschiedenen Ebenen der Beeinträchtigung: nämlich bezogen auf Körperstrukturen und -funktionen, wie eine Sehschädigung, auf Aktivitäten, wie Sehen, Lesen, sich Orientieren, sich Bewegen, und auf Teilhabe, wie Einbeziehung in Gerätegebrauch, Mobilität oder die Kommunikation. Zugleich wird der Blick gelenkt auf die Wechselwirkungen der Person- und Umweltfaktoren mit Teilhabe- und Behinderungserfahrungen im Lebensverlauf sowie auf Zugehörigkeit und Zugehörigkeitsgefühl.
} 
wissenschaftliche Technik damit umzugehen ist Diversity Mainstreaming. Es bedeutet, über Fächergrenzen und Leistungssysteme hinweg in einer ethnisch, sozial und kulturell vielfältigen Gesellschaft zu prüfen, wo und wie gerechte Teilhabemöglichkeiten auffindbar sind bzw. entstehen können und wo hier Grenzen liegen. Die Wissenschaft kann zugleich ihre Fitness prüfen für die sich überschneidenden Formen von Unterschiedlichkeit, also für ,super-diversity“ (Vertovec 2007), die nicht mehr nur reduziert (oder übersehen) werden muss.

Aus der Zusammenschau des kulturell geprägten Zugangs über die Human Rights verbunden mit der analytischen Frage nach der Aufmerksamkeit für Human Resource ergeben sich Perspektiven auf eventuell vorliegende oder drohende Teilhabestörungen. Wie sich hier Teilhabeforschung einsetzen kann, ist vor allem eine Zukunftsfrage, hat also Potenzial zum Ausblick.

\subsection{Die Gemeinschaftsperspektive: Teilhabe inklusive I Human Relation Approach}

Diversity-Forschung und Inklusionsprogrammatiken treffen sich auf „,schlüpfrigem Gelände"; manche reden von einer empirischen Zwiebel (Ragins und Gonzales 2003, S. 133), die durch ihre Vielschichtigkeit faszinieren mag, gewiss aber Forschung herausfordert. Zugleich stehen die folgenden bereits reflektierten und ausgehandelten Perspektiven zur Verfügung:

- die Aufmerksamkeit auf die Person und in ihr liegende Merkmale, meist am Beispiel der sogenannten Big 8 (race, gender, ethnicity/nationality, organizational role/function, age, sexual orientation, mental/physical ability, religion),

- die Akzentuierung der Unterschiede über Machtverhältnisse, also Dominanzstrukturen, die bereits eingeschrieben sind in Kultur und ihre Organisation, als Maß der Abweichungen (doing diversity als soziale Dimension),

- relationale Wirkzusammenhänge in Gemeinschaften, die dann beispielsweise der Gruppe der definierten Behinderten Plätze zuweisen.

Wenn Teilhabeforschung ihrem Aufklärungsanliegen folgt, hat sie also Möglichkeiten, Anpassungszwänge aufzudecken, um inklusivere Bedingungen für eine Selbst-Beheimatung und Lebensführung ohne Anpassungszwang anzuregen. Dies kreuzt sich mit Gender- und Diversitätsforschung, die einfordern:

Not to celebrate difference as such but to establish more inclusive conditions of sheltering and maintaining life that resists models of assimiliation (Butler 2004, S. 4).

Auch ein Pakt mit einer neuen agilen Gesundheitsforschung, die dem CapabilityApproach folgt (Bertmann 2018; Mitchell et al. 2017), ist denkbar. Mit erprobten Maßnahmen und über die neue Aufmerksamkeit für Diversität und Inklusion können Einbeziehung, Wertschätzung und Anerkennung in laufende Aushandlungsprozesse etwa mit Blick auf Prävention, Rehabilitation und Gesundheitsförderung eingeschrieben werden. Dazu zählen ebenso strukturelle Vorgaben, die mit Sensibilisierungs- (Trainings) oder Fördermaßnahmen (Mentoring) anzureichern wären. Eine nimmermüde Mainstreaming Strategie soll gelingen zwischen Zugehörigkeit 
(belonging) und Wertschätzung. Wachsende Diversitäts- und Inklusionskompetenz spielen dabei eine zentrale Rolle.

Die bekannte Anwältin und Aktivistin, Vernā Myers, macht bildlich aufmerksam: „Diversity is being invited to the party; inclusion is being asked to dance."12

Als Voraussetzung ist eine Perspektive der unbedingten Zugehörigkeit zentral, weil sie wertschätzend wirkt. Zugehörigkeit und human bonding, als Verflechtungen aus emotionaler Bindung, Zuneigung und Vertrauen, reichen weit über Rechtsansprüche hinaus. Wertschätzung zu erfahren, wird als Bedürfnis ebenso basal wie Essen, Trinken und Schlafen eingeordnet: ,in fact, social bonding is as vital to human survival as food and drink“ (Hazan und Campa 2013).

Zugleich besteht die Versuchung fort, Menschen an einer als fraglos gegeben erscheinenden Normalität zu messen, auszurichten und an bewährte Erwartungen und diese fiktiven Normen anzupassen. Der Inklusionsgedanke zeigt in eine umgekehrte Richtung, nämlich Umgebungen so zu gestalten, dass sie Kompetenzen und Selbstbestimmung jeweils nicht verstellen, sondern zutage fördern. Dies entspricht der Utopie einer Transformation zur inklusiven Gesellschaft, die nur in Gemeinschaft vorstellbar ist. Hier könnte Forschung ihre Kerntriebkraft Fantasie entfalten. Ein Beispiel gibt das Programm IDEA: ${ }^{13}$

- Inclusion: An individual or group sense of belonging as a valued member of society

- Diversity: Openness and an awareness of diversity

- Equity: Fair and just outcomes for all to achieve the full intellectual and professional potential

- Access: Opportunities to fully participate in society.

Abb. 2 Das 3HR-Modell: Human Rights, Human Resource, Human Relations

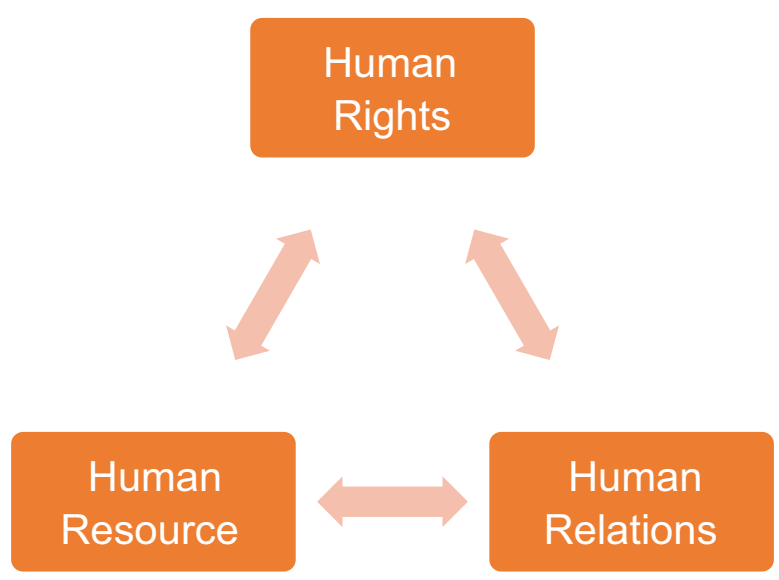
Rights

12 Myers ist eine in Havard qualifizierte Anwältin und Diversitätsaktivistin aus der Gemeinschaft der African Americans. https://www.ted.com/speakers/verna_myers. Zugegriffen: 20. September 2020.

13 https://www.un.org/esa/socdev/documents/compilation-brochure.pdf. Zugegriffen: 20. September 2020 . 
Aus Inklusion mit der Botschaft der Zugehörigkeit und Wertschätzung an Individuen und Gruppen, Diversität als Offenheit und Bewusstsein für Vielfalt, Gerechtigkeit als faire und angemessene Bedingungen für alle, lässt sich Teilhabeforschung komponieren und realisieren. Denn im Dreiklang von Human Rights, Human Resource und Human Relations (3HR-Modell, wie in Abb. 2 skizziert) lässt sich prüfen, welche Personen und Gruppen mögliche volle intellektuelle und professionelle Potenziale erreichen und ob Zugang zur vollen Teilhabe an der Gesellschaft nicht nur als Anspruch, sondern auch als Chance besteht.

So entsteht eine neue Sichtweise auf Behinderung. Über die Zulassung zur Gemeinschaft menschlicher Vielfalt und Mitsprache hinaus geht es um die selbstverständliche Anerkennung der Relevanz individueller Anliegen. Die Strategie ist nicht Umverteilung, sondern eine Politik der Anerkennung von Gleichwertigkeit bei Verschiedenheit.

Damit kann sich Forschung beherzt auf die vielbefahrene Kreuzung des Lebens wagen und verschiedene Teilhabeanliegen intersektional begleiten. Es geht nicht mehr alleine um spezifische Aspekte derer am Rande der Gesellschaft, weil alle Gesellschaftsmitglieder eingeschlossen sind. Teilhabeforschung kann hierbei Kräfte einbringen und innovativ wirken durch die genannten Transformationspotenziale, verbunden mit Phantasie und Handlungsorientierung.

\section{Ausblick - Placet experiri}

Placet experiri steht für „Aufnahmelust“, „Loslösung von festen Verhältnissen“, Sensibilisierung von „Wahrnehmungen“ (Pikulik 2011, S. 94 f.), kurz einen Zauber mit neuer Weltsicht, den Thomas Mann (1924) im Roman „Der Zauberberg“ darstellt. Grenzen des Gegebenen werden in Gedankenspielen und auch in Handlungen überschritten. Diese Verlockung können experimentierfreudige Forscherinnen und Forscher in Handlungsfeldern der Teilhabe erspüren. Entsprechende Einladungen zur Aufnahmelust haben Schütz und Luckmann (1975, S. 267f.) bereits vor einem halben Jahrhundert ausgesprochen:

Jedwedes Entwerfen innerhalb der Welt des Wirkens ist ... ein Phantasieren und impliziert zusätzlich eine Art theoretischer Kontemplation, wenn auch nicht notwendig eine wissenschaftliche Einstellung.

Explizit sind so alle zum Tanz aufgefordert (siehe 3.3) und können sich mit ihrer jeweiligen Expertise in eine ,teilhabeversessene“ Sozialforschung einbringen. Zur theoretischen Kontemplation kommen Hilfen der Vorstellungskraft, die womöglich beherztes kreatives Handeln hervorbringen. Statt fertige Lösungen finden sich derzeit zunächst Anstöße zur Steuerung. Das kann auch Forschungsexpertise mit Blick auf soziale Probleme locken, auf ,die Plätze, wo jeder Mann, jede Frau und jedes Kind gleiche Rechte, gleiche Chancen und gleiche Würde ohne Diskriminierung sucht" wie eingangs beschrieben.

Auch Forschung hat ihre Zeit und ihren Raum. Dies wurde in der Sozialpolitik (zu) lange Zeit vernachlässigt, mahnt Becker (2013, S. 11). Wenn nun alle geladen sind mitzutanzen, könnte „Teilhabe im Sozialraum“ auch neu Karriere machen und 
die Kommunen verdienen zukünftig in besonderer Weise wissenschaftliche Aufmerksamkeit, eingebettet in und verflochten mit staatlichen und länderspezifischen Rahmungen. Denn Kommunen sollen umfangreiche Daseinsvorsorge garantieren. Deren Freiheit der Entscheidung ist zugleich eingebunden in Pflichtaufgaben und gesetzliche Vorgaben bzw. Standards. Statt gleiche Teilhabe für alle zu ermöglichen, bieten sozialräumliche Realsituationen aber faktisch erhebliche Ungleichbehandlungen (Henkel 2010, S. 212). Der Staat ist bedingt wirksam als Vereinheitlicher, dies gilt analog für örtliche und überörtliche Sozialleistungsträger. Nun erfordert die angemessene Erfüllung sozialpolitischer Aufgaben ständig steigende finanzielle Mittel, passgenaue Infrastrukturen und entsprechende Aufmerksamkeiten. Hierzu sind Finanzmittel erforderlich, die weit über die kommunale Leistungsfähigkeit hinausreichen, aber mit einem „Aufgabenübertragungsverbot“"(Art. 84 Abs. 1 S. 7 GG) werden „Übergriffe“ staatlicherseits eingedämmt. Das Zusammenspiel in der Daseinsvorsorge ist somit voraussetzungsreich und kompliziert; Teilhabechancen für alle gleichermaßen zu eröffnen, ist herausfordernd. Auch hier wäre Teilhabeforschung eine potenzielle Partnerin aller Beteiligten, wenn Teilhabe ein Gemeingut ist (Ostrom und Helfrich 2011). Neue Haltungen zu den „,commons“, neue Gemeinschaften - mit partizipativen Methoden und Aufmerksamkeit für Solidarität und Chancengerechtigkeit (von Unger 2014) - werden durch ein tiefes und reflektiertes entsprechendes Forschungsverständnis an Kraft gewinnen und Diversitäts- und Inklusionskompetenz verbinden.

Gegenwartsanalysen finden sich stets. Eine das Ende des vergangenen Jahrtausends prägende soziologische Deutung ist die eingangs genannte unaufhaltsame und unumkehrbare Individualisierung mit Ansprüchen und Anforderungen an persönlich gestaltbare und gelingende Lebensführung in Selbstbestimmung und Risiken für Gemeinschaft (Beck 1986). Dem könnte ein Zeitalter der Gemeinschaften folgen. Mögliche Treiber sind Aufmerksamkeit für Diversität (Human Resource: Power), Bewusstheit für Intersektionalität und eingeschriebene Wechselwirkungen (Human Relations: Inclusiveness, Individualität und Zusammenhalt, Interdependenz, Gemeingüter) und Inklusion als Zielorientierung (Human Rights: Shelter, Verlässlichkeit). Für innovationswillige Forschung lohnt es sich, solchen Verlockungen zu folgen und die Teilhabebrille aufzusetzen. Mit der Teilhabeidee schärft sich womöglich die Einsichtstiefe. Möglichkeiten von Neudeutungen zwischen Diversitätsformeln und Inklusionsutopien werden sichtbar, die weiterreichen als die Behandlung der ,Behinderungsfrage“.

Danksagung Die Autorin dankt dem Max-Planck-Institut für Sozialrecht und Sozialpolitik, München, für eine Dekade fruchtbarer Zusammenarbeit als Fellow. Wertschätzung wirkt.

Funding Open Access funding enabled and organized by Projekt DEAL.

Open Access Dieser Artikel wird unter der Creative Commons Namensnennung 4.0 International Lizenz veröffentlicht, welche die Nutzung, Vervielfältigung, Bearbeitung, Verbreitung und Wiedergabe in jeglichem Medium und Format erlaubt, sofern Sie den/die ursprünglichen Autor(en) und die Quelle ordnungsgemäß nennen, einen Link zur Creative Commons Lizenz beifügen und angeben, ob Änderungen vorgenommen wurden.

Die in diesem Artikel enthaltenen Bilder und sonstiges Drittmaterial unterliegen ebenfalls der genannten Creative Commons Lizenz, sofern sich aus der Abbildungslegende nichts anderes ergibt. Sofern das betref- 
fende Material nicht unter der genannten Creative Commons Lizenz steht und die betreffende Handlung nicht nach gesetzlichen Vorschriften erlaubt ist, ist für die oben aufgeführten Weiterverwendungen des Materials die Einwilligung des jeweiligen Rechteinhabers einzuholen.

Weitere Details zur Lizenz entnehmen Sie bitte der Lizenzinformation auf http://creativecommons.org/ licenses/by/4.0/deed.de.

\section{Literatur}

Antonovsky, Aaron. 1997. Salutogenese. Zur Entmystifizierung der Gesundheit. Tübingen: dgvt.

Beck, Ulrich. 1986. Risikogesellschaft. Auf dem Weg in eine andere Moderne. Frankfurt: Suhrkamp.

Becker, Ulrich. 2013. Aufgaben und Handlungsspielräume der Kommunen als Ort der Inklusion. In Inklusion und Sozialraum. Behindertenrecht und Behindertenpolitik in der Kommune, Hrsg. Ulrich Becker, Elisabeth Wacker, und Minou Banafsche, 11-24. Baden-Baden: Nomos.

Becker, Ulrich, Elisabeth Wacker, und Minou Banafsche (Hrsg.). 2013. Inklusion und Sozialraum. Behindertenrecht und Behindertenpolitik in der Kommune. Baden-Baden: Nomos.

Becker, Ulrich, Elisabeth Wacker, und Minou Banafsche (Hrsg.). 2015. Homo faber disabilis? Teilhabe am Erwerbsleben. Baden-Baden: Nomos.

Bendel, Klaus. 1999. Behinderung als zugeschriebenes Kompetenzdefizit von Akteuren. Zur sozialen Konstruktion einer Lebenslage. Zeitschrift für Soziologie 28(4):301-310.

Berger, Peter L., und Thomas Luckmann. 1969. Die gesellschaftliche Konstruktion der Wirklichkeit. Eine Theorie der Wissenssoziologie. Frankfurt: Fischer.

Bertmann, Isabella. 2018. Taking well-being and quality of life for granted? An empirical study on social protection and disability in South Africa. Wiesbaden: Springer VS.

Butler, Judith. 2004. Undoing gender. New York: Routledge.

Crenshaw, Kimberlé W. 1989. Demarginalizing the intersection of race and sex: a black feminist critique of Antidiscrimination doctrine. The University of Chicago Legal Forum 1(1):139-167.

Crenshaw, Kimberlé W. 2010. Die Intersektion von „Rasse“ und Geschlecht demarginalisieren: Eine schwarze feministische Kritik am Antidiskriminierungsrecht, der feministischen Theorie und der antirassistischen Politik. In Fokus Intersektionalität - Bewegung und Verortung eines vielschichtigen Konzeptes, Hrsg. Helma Lutz, Maria Teresa Herrera Vivar, und Linda Supik, 33-54. Wiesbaden: VS.

Demant, Luisa. 2017. Teilhabe an Bildung. Beratung und professionelles Handeln. Wiesbaden: Springer VS.

Die Redaktion. 2006. Die Soziologie sozialer Probleme in der Krise? 30 Jahre Sektion „Soziale Probleme und Soziale Kontrolle“. Eine Diskussion zum Stand der Dinge. Soziale Probleme 17(1):5-8.

Dobusch, Laura. 2015. Diversity Limited. Inklusion, Exklusion und Grenzziehungen mittels Praktiken des Diversity Management. Wiesbaden: Springer VS.

Dobusch, Laura. 2017. Von der Diversitäts- zur Inklusionsforschung? zeitschrift für diversitätsforschung und -management diversitatis 2(1):59-63.

Ferdman, Bernardo M. 2014. The practise of inclusion in diverse organizations. In Diversity at work: the practice of inclusion, Hrsg. Bernardo M. Ferdman, Barbara R. Deane, 3-54. San Francisco: JosseyBass.

Finzen, Asmus. 2018. Normalität - Die ungezähmte Kategorie in Psychiatrie und Gesellschaft. Köln: Psychiatrie-Verlag.

Finzen, Asmus. 2009. Psychiatrie und Soziologie. Eine Einladung. http://www.finzen.de/pdf-dateien/ soziologie.pdf. Zugegriffen: 20. Sept. 2020.

Gardenswartz, Lee, und Anita Rowe. 2003. Internal dimensions and external dimensions. https://www. gardenswartzrowe.com/diversity.doc. Zugegriffen: 6. Sept. 2019.

Geißler, Rainer. 1994. Die pluralisierte Schichtstruktur der modernen Gesellschaft: zur aktuellen Bedeutung des Schichtbegriffs. In Soziale Schichtung und Lebenschancen in Deutschland, 2. Aufl., Hrsg. Rainer Geißler, 6-36. Stuttgart: Enke.

Goffman, Erving. 1967. Stigma. Über Techniken der Bewältigung beschädigter Identität. Frankfurt: Suhrkamp.

Gottburgsen, Anja, und Christiane Gross. 2012. Welchen Beitrag leistet die „Intersektionalität“ zur Klärung von Kompetenzunterschieden bei Jugendlichen. In Soziologische Bildungsforschung. In Kölner Zeitschrift für Soziologie und Sozialpsychologie (Sonderheft 52), Hrsg. Rolf Becker, Heike Solga, 86-110. Wiesbaden: Springer VS. 
Groenemeyer, Axel. 2006. Gesellschaftspolitische Relevanz und soziologische Reputation. Eine kleine Geschichte über 30 Jahre Soziologie sozialer Probleme. Soziale Probleme 17(1):9-19.

Groenemeyer, Axel. 2014. Soziale Praxis - Institutionen - Diskurse - Erfahrung. Behinderung im Problematisierungsprozess. In Diversität \& Inklusion. Umgang mit Vielfalt und Verschiedenheit bei Beeinträchtigung und Behinderung Soziale Probleme. Zeitschrift für Soziale Probleme und Soziale Kontrolle 25., Hrsg. Elisabeth Wacker, Axel Groenemeyer, 150-172.

Haake, Gianna. 2018. Vorkenntnisse und Zeitbudget als Kerndimensionen des hochschulischen Diversity Managements - Theoretische Perspektiven und empirische Erprobung. zeitschrift für diversitätsforschung und -management diversitatis 3(1):21-33.

Hall, Edward T. 1976. Beyond culture. New York: Anchor Books.

Hazan, Cindy, und Mary I. Campa (Hrsg.). 2013. Human bonding: the science of affectional ties. Guilford Press. https://psycnet.apa.org/record/2013-26053-000. Zugegriffen: 27. Sept. 2020.

Henkel, Jörg. 2010. Die Kommunalisierung von Staatsaufgaben. Eine Herausforderung für die kommunale Selbstverwaltung und ihre Dogmatik. Stuttgart: Richard Boorberg.

Hinz, Renate. 2009. Bildungspolitische Analyse. In Heterogenität in der Grundschule. Den pädagogischen Alltag erfolgreich bewältigen, Hrsg. Renate Hinz, Renate Walthes, 16-31. Weinheim, Basel: Beltz.

Kaufmann, Franz-Xaver. 2012. Konzept und Formen sozialer Intervention. In Handbuch soziale Probleme, 2. Aufl., Bd. 2, Hrsg. Günter Albrecht, Axel Groenemeyer, 1285-1305. Wiesbaden: Springer VS.

Kocks, Klaus. 2001. Glanz und Elend der PR. Zur praktischen Philosophie der Öffentlichkeitsarbeit. Wiesbaden: Westdeutscher Verlag.

Krell, Gertraude. 2014. „Wahrheitsspiele“: Diversity versus oder inklusive Intersektionalität? In Diversität \& Inklusion. Umgang mit Vielfalt und Verschiedenheit bei Beeinträchtigung und Behinderung Soziale Probleme. Zeitschrift für Soziale Probleme und Soziale Kontrolle 25., Hrsg. Elisabeth Wacker, Axel Groenemeyer, 194-208.

Kuhn, Thomas S. 2009. Die Struktur wissenschaftlicher Revolutionen, 2. Aufl., Frankfurt: Suhrkamp.

Lewin, Kurt. 1948. Aktionsforschung und Minderheitenprobleme. In Die Lösung sozialer Konflikte, Hrsg. Kurt Lewin, 278-298. Bad-Nauheim: Christian.

Löw, Martina. 2014. Vielfalt und Repräsentation. Über den Bedeutungsverlust der symbolischen Mitte. In Vielfalt und Zusammenhalt. Verhandlungen des 36. Kongresses der Deutschen Gesellschaft für Soziologie in Bochum und Dortmund 2012, Hrsg. Martina Löw, 27-37. Frankfurt: Campus. Teil 1.

Luhmann, Niklas. 1980. Gesellschaftsstruktur und Semantik. Frankfurt: Suhrkamp.

Luhmann, Niklas. 1995. Die gesellschaftliche Differenzierung und das Individuum. In Soziologische Aufklärung 6. Die Soziologie und der Mensch, Hrsg. Niklas Luhmann, 125-141. Opladen: Westdeutscher Verlag.

Luhmann, Niklas. 1997. Die Gesellschaft der Gesellschaft. Frankfurt: Suhrkamp.

Mann, Thomas. 1924. Der Zauberberg. Berlin: Fischer.

Marshall, Thomas H. 1950. Citizenship and social class. Cambridge: University Press.

Mead, Georg Herbert. 1934. Geist, Identität und Gesellschaft aus der Sicht des Sozialbehaviorismus. Frankfurt: Suhrkamp.

Mecheril, Paul, und Melanie Plößer. 2018. Diversity und Soziale Arbeit. In Handbuch Soziale Arbeit, 6. Aufl., Hrsg. Hans-Uwe Otto, Hans Thiersch, Rainer Treptow, und Holger Ziegler, 283-292. München: Reinhardt.

Mensi-Klarbach, Heike, Günther Vedder, und Andrea Wolffram. 2017. Editorial Schwerpunktthema: Vielfältige Differenzlinien in der Diversitätsforschung. zeitschrift für diversitätsforschung und-management diversitatis 2(1):4-6.

Mitchell, Paul Mark, Tracy E. Roberts, M. Barton Pelham, und Joanna Coast. 2017. Applications of the capability approach in the health field: a literature review. Soc Indic Res 133:345-371. https://doi.org/ 10.1007/s11205-016-1356-8.

Nassehi, Armin. 2014. Hat die Soziologie einen sozialen Bias? In Vielfalt und Zusammenhalt. Verhandlungen des 36. Kongresses der Deutschen Gesellschaft für Soziologie in Bochum und Dortmund 2012, Hrsg. Martina Löw, 57-68. Frankfurt: Campus. Teilband 1.

Ostrom, Elinor, und Silke Helfrich (Hrsg.). 2011. Was mehr wird, wenn wir teilen. Vom gesellschaftlichen Wert der Gemeingüter. München: Oekom.

Oswick, Cliff, und Mike Noon. 2014. Discourses of diversity, equality and inclusion: trechant formulations or transient fashions? British Journal of Management 25(1):23-39.

Parsons, Talcott. 1972. Das System moderner Gesellschaften. München: Juventa.

Pikulik, Lothar. 2011. Placet experiri. In Thomas Mann. Der Künstler als Abenteurer, 94-100. Paderborn: mentis. https://doi.org/10.30965/9783957439666_011. 
Ragins, Belle Rose, und Jorge A. Gonzalez. 2003. Organizational diversity: Getting a grip on a slippery construct. In Organizational behavior: The State of the Science, Hrsg. Jerald Greenberg, 125-163. Mahwah: Lawrence Erlbaum.

Rosenthal, Klaus. 2017. Der Blickwechsel in der Wissenschaft. Weilerswist-Metternich: Velbrück Wissenschaft.

Schäfers, Markus, Viviane Schachler, Ulrich Schneekloth, Elisabeth Wacker, und Ekaterina Zeiler. 2016. Pretest Befragung in Einrichtungen. Abschlussbericht zum 31.05.2016. http://www.bmas.de/ DE/Service/Medien/Publikationen/Forschungsberichte/Forschungsberichte-Teilhabe/fb471-pretestbefragung-ineinrichtungen.html.. Zugegriffen: 26. Juni 2020.

Schöer, Hubertus. 2015. Diversity und interkulturelle Öffnung. Sozialmagazin 9(10):30-36.

Schönig, Werner. 2019. Typologie und Klassifikation in Sozialer Arbeit und Sozialpolitik. Ambivalenz und kritische Nutzung von Ordnungsschemata sozialer Probleme. Baden-Baden: Nomos.

Schütz, Alfred, und Thomas Luckmann. 1975. Strukturen der Lebenswelt. Neuwied: Luchterhand.

Sen, Amartya. 1985. Commodities and Capabilities. Amsterdam: North Holland.

Sen, Amartya. 2007. Ökonomie für den Menschen. München: dtv.

Sen, Amartya. 2010. Die Idee der Gerechtigkeit. München: Beck.

von Unger, Hella. 2014. Partizipative Forschung. Einführung in die Forschungspraxis. Wiesbaden: Springer VS.

United Nations. 2006. Convention on the rights of persons with disabilities. http://www.un.org/disabilities/ documents/convention/convoptprot-e.pdf. Zugegriffen: 23. Juli 2020.

Vertovec, Steven. 2007. Super-diversity and its implications. Ethnic and Racial Studies 30(6):1024-1054. https://doi.org/10.1080/01419870701599465.

Wacker, Elisabeth. 2013. Überall und nirgendwo - „Disability Mainstreaming“ im kommunalen Lebensraum und Sozialraumorientierung als Transformationskonzept. In Inklusion und Sozialraum. Behindertenrecht und Behindertenpolitik in der Kommune, Hrsg. Ulrich Becker, Elisabeth Wacker, und Minou Banafsche, 25-45. Baden-Baden: Nomos.

Wacker, Elisabeth. 2014. Verwobene Behinderungsprobleme. Diversität und Inklusivität als Spagat und Zwickmühle. In Diversität \& Inklusion. Umgang mit Vielfalt und Verschiedenheit bei Beeinträchtigung und Behinderung Soziale Probleme. Zeitschrift für Soziale Probleme und Soziale Kontrolle 25., Hrsg. Elisabeth Wacker, Axel Groenemeyer, 231-267.

Wacker, Elisabeth. 2016. Beeinträchtigung - Behinderung - Teilhabe für alle. Neue Berichterstattung der Bundesregierung zur Teilhabe im Lichte der Behindertenrechtskonvention der Vereinten Nationen. Behinderung und Gesellschaftliche Teilhabe. Bundesgesundheitsblatt 59(9):1093-1102. https://doi. org/10.1007/s00103-016-2397-5.

Wacker, Elisabeth, und Axel Groenemeyer (Hrsg.). 2014. Diversität \& Inklusion. Umgang mit Vielfalt und Verschiedenheit bei Beeinträchtigung und Behinderung. Soziale Probleme. Zeitschrift für Soziale Probleme und Soziale Kontrolle 25.

Wacker, Elisabeth, Ulrich Becker, und Eva Nachtschatt. 2020. Wem gehört die Teilhabe? Nutzen, Bereitstellung und Verfügbarkeit von Teilhabe als Allgemeingut. Baden-Baden: Nomos. im Druck.

Wacker, Elisabeth, Gudrun Wansing, und Markus Schäfers. 2009. Personenbezogene Unterstützung und Lebensqualität. Teilhabe mit einem Persönlichen Budget, 2. Aufl., Wiesbaden: VS.

Wacker, Elisabeth. 2019. Teilhabeforschung als Transformationsforschung. 2tf: Teilhabe for the future: Key Note 27.09.2019. https://www.reha-recht.de/fachbeitraege/beitrag/artikel/beitrag-d2-2020. Zugegriffen: 22. Juni 2020.

Waldschmidt, Anne. 2006. Soziales Problem oder kulturelle Differenz? Zur Geschichte von „Behinderung“ aus der Sicht der „Disability Studies“. traverse 13(3):31-46.

Waldschmidt, Anne. 2014. Macht der Differenz. Perspektiven der Disability Studies auf Diversität, Intersektionalität und soziale Ungleichheit. In Diversität \& Inklusion. Umgang mit Vielfalt und Verschiedenheit bei Beeinträchtigung und Behinderung Soziale Probleme. Zeitschrift für Soziale Probleme und Soziale Kontrolle 25., Hrsg. Elisabeth Wacker, Axel Groenemeyer, 173-193.

Walgenbach, Katharina. 2018. Intersektionalität und Diversity - zwei kompatible Paradigmen? zeitschrift für diversitätsforschung und-management diversitatis 3(1):34-48.

Walthes, Renate, und Ronald Hitzler. 2018. Begleitwort. In Zwischen Sehen und Nicht-Sehen. Eine wahrnehmungs- und lebensweltanalytische Ethnographie zur Situation von Menschen mit Sehbeeinträchtigung im Alter, Hrsg. Carsten Bender, Marion Schnurnberger, 9-12. Weinheim, Basel: Beltz Juventa.

Weisser, Gerhard. 1978. Beiträge zur Gesellschaftspolitik. Göttingen: Schwartz.

WHO - World Health Organization. 2001. International classification of functioning, disability and health. Geneve: WHO. 